\title{
REVISION OF MILLETTIA SECTION TRUNCATICALYCES (LEGUMINOSAE-PAPILIONOIDEAE)
}

\author{
NEDUVOTO MOLLEL \& FRITS ADEMA \\ Nationaal Herbarium Nederland, Universiteit Leiden branch, \\ P.O. Box 9514, 2300 RA Leiden, The Netherlands
}

\section{SUMMARY}

A revision of Millettia sect. Truncaticalyces is presented. Five species are recognized. A key to the species and an identification list are included.

Key words: Millettia, section Truncaticalyces, Africa.

\section{INTRODUCTION}

Millettia was described by Wight \& Arnott (1834: 263) to accommodate two new species: M. rubiginosa Wight \& Arn. and M. splendens Wight \& Arn. Since then many species have been added to this genus. Dunn (1912) accepted 127 species in 15 sections. Geesink $(1981,1984)$ transferred several sections to other genera and united Pongamia Vent. with Millettia sect. Fragiliflorae. In the following treatment sect. Truncaticalyces of the genus will be revised. The species of sect. Truncaticalyces are found in various habitats in Central and West tropical Africa. Some Millettia species are extensively grown in tropical Africa as shade and ornamental trees along roads. Besides timber and ornamental flowers, the genus in general provides other useful products. Certain species in both Africa and Asia are known to native fishermen for a chemical substance (rotenone) in wood, fruits, etc. which has properties to stupefy or kill fish. For example, M. ferruginea (Hochst.) Baker is widely known as a fish poison. Similar compounds are found in Derris, Lonchocarpus and Paraderris. The stems of many Millettia species are used in Chinese herbal medicines.

\section{TAXONOMIC TREATMENT}

\section{MILLETTIA}

Millettia Wight \& Arn. (1834) 263, nom. cons.; Benth. (1852) 247; Baker (1879) 104; Taub. (1894) 270; Dunn (1912) 123; Baker f. (1929) 216; O.N. Allen \& E.K. Allen (1981) 435; R. Geesink (1981) 257; (1984) 102. - Millettia Wight \& Arn. sect. Typicae Dunn (1912) 134. - Lectotype (Hutchinson, 1964): Millettia rubiginosa Wight \& Arn.

Trees, shrubs or lianas, or rarely semi-herbaceous plants with a woody rootstock. Leaves imparipinnate, rarely pinnately trifoliolate; petiole with a pulvinus; stipules present, caducous; stipellae present or absent. Leaflets entire, opposite to alternate, secondary 
nerves not reaching the margin. Inflorescences axillary or terminal, racemose, often pseudoracemes. Bracts present, caducous; bracteoles present, often caducous. Calyx truncate or with short teeth (up to $1.5 \mathrm{~mm}$ long), or divided up to two-thirds of its length. Corolla: standard with or without basal callosities, rarely with auricles; wings adhering to the keel petals; keel petals slightly shorter than the wings, usually with lateral pockets. Stamens mon- or diadelphous, in the former case with basal fenestrae; filaments not widened at the apex; anthers all equal, fertile, basi- or dorsifixed, usually glabrous. Disc inconspicuous to annular, or 10-lobed and adnate to the hypanthium. Ovary (sub) sessile to stipitate, with (2 or) 3-11 ovules; style straight or incurved at apex; stigma terminal, small, discoid. Pods strap- or 'club'-shaped, dehiscent. Seeds flattened ellipsoid, hilum central.

Distribution - Circa 200 species in tropical and subtropical Africa, Asia into the Pacific.

\section{Section TRUNCATICALYCES}

Section Truncaticalyces Dunn (1912) 137; Baker f. (1929) 221. - Lectotype (here designated): Millettia macrophylla Benth.

Trees or lianas. Stems lenticellate, hollow or not. Leaflets opposite. Stipellae absent. Inflorescences axillary, pseudoracemes. Calyx truncate or with up to $1.5 \mathrm{~mm}$ long, broad triangular teeth. Petals glabrous or sericeous outside at the apex; standard without callosities or auricles. Ovules $4-8$.

Note - For a key to the sections see Dunn (1912) and Baker (1929).

\section{KEY TO THE SPECIES}

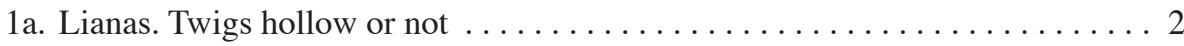

b. Trees. Twigs hollow . . . . . . . . . . . . . . . . . . . . 3

2a. Twigs hollow. Leaves with 5-8 pairs of leaflets. Lower surface of leaflets velutinous, upper surface sericeous. Standard golden sericeous at apical margins. Keel petals outside golden sericeous in apical part . . . . . . . M. aboensis

b. Twigs not hollow. Leaves with 2-6 pairs of leaflets. Lower surface of leaflets scabrous, upper surface glabrous. Standard glabrous. Keel petals glabrous . . . . .

4. M. psilopetala

3a. Lower surface of leaflets sericeous or thinly strigose. Standard outside golden sericeous in apical part. Keel petals outside golden sericeous in apical part. Stamens

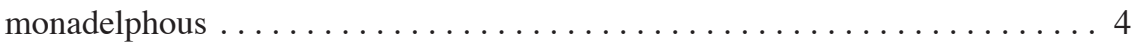

b. Lower surface of leaflets with few appressed, scattered hairs. Standard glabrous. Keel petals glabrous. Stamens diadelphous . . . . . . . . 5. M. thonneri

4a. Leaves with 7-11 pairs of leaflets. Lower surface of leaflets sericeous . . . . . . .

3. M. mannii

b. Leaves with 2-6 pairs of leaflets. Lower surface of leaflets strigose . . . . . . . 
1. Millettia aboensis (Hook.f.) Baker - Fig. 1, 2a, b, 3a-e

Millettia aboensis (Hook.f.) Baker (1871) 130; Dunn (1912) 214; Baker f. (1929) 236; Hepper (1958) 505. - Millettia macrophylla Benth. var. aboensis Hook.f. in Hook.f. \& Benth. (1849) 319. - Lectotype (here designated): Ansell 360 (holo K; iso K), Nigeria, 1841.

Lianas up to $9 \mathrm{~m}$ high. Twigs hollow, brown velutinous. Leaves with 5-8 pairs opposite leaflets. Petiole $3.5-8 \mathrm{~cm}$ long, brown velutinous; rachis $13-20 \mathrm{~cm}$ long, brown velutinous; pulvinus $0.5-1 \mathrm{~cm}$ long, strigose. Stipules linear-ovate, $6-9$ by $1-3 \mathrm{~mm}$, sericeous. Leaflets: terminal narrowly obovate, $8.5-15$ by $3-5 \mathrm{~cm}$, index $1.9-3.4$, base cuneate, apex acuminate, acumen $0.2-0.6 \mathrm{~cm}$ long, upper surface brown strigose, lower surface brown velutinous, midrib and nerves flat above, main nerves 9 or 10 pairs per side, $8-15 \mathrm{~mm}$ apart, nervation reticulate; lateral mostly as the terminal, (broadly) ovate, $3.5-9$ by $2.2-4.5 \mathrm{~cm}$, index $1.4-3$, base broadly cuneate or rounded, main nerves 6-9 per side, $6-9 \mathrm{~mm}$ apart; pulvinus $0.4-0.7 \mathrm{~cm}$ long, brown strigose. Inflorescences $16-54 \mathrm{~cm}$ long, brown strigose, peduncle up to $16 \mathrm{~cm}$ long, brown strigose; brachyblasts 15 to many, c. 12-flowered. Bracts to the inflorescence linear-ovate, c. 6 by 2 $\mathrm{mm}$; other bracts \pm similar, triangular, c. 4 by $2 \mathrm{~mm}$, outside brown velutinous, inside glabrous. Pedicels $6-10 \mathrm{~mm}$ long, brown velutinous. Bracteoles caducous. Calyx 7-8 $\mathrm{mm}$ long, truncate, outside brown velutinous, inside glabrous. Corolla purple. Standard: claw 3-9 $\mathrm{mm}$ long; blade ovate to broadly obovate, $15-24$ by $10-20 \mathrm{~mm}$, apex emarginate, mostly glabrous, golden sericeous at apical margins. Wings: claw 6-12 $\mathrm{mm}$ long; blade obliquely obovate, $13-18$ by $4-5 \mathrm{~mm}$, upper auricle conspicuous, apex obtuse, glabrous. Keel petals: claw 6-12 mm long; blade hatchet-shaped, 12-16 by $5-6 \mathrm{~mm}$, upper auricle inconspicuous, lateral pocket $5-10 \mathrm{~mm}$ long, apex obtuse, mostly glabrous, outside golden sericeous in apical part. Stamens monadelphous, tube 12-23 mm long, glabrous, free part of filaments 5-7 mm long, curved, glabrous; anthers $1-1.3$ by $0.6-1 \mathrm{~mm}$, glabrous. Ovary $14-22 \mathrm{~mm}$ long, sericeous; ovules 6 ; style $4-8$ $\mathrm{mm}$ long, strigose near the base, glabrous above, curved. Pods strap- or 'club'-shaped, flattened, $9-15$ by $0.8-2.2 \mathrm{~cm}$, dark brown velutinous.

Distribution - Nigeria, Cameroon.

Habitat \& Ecology - Secondary forest. Altitude 50 m (from 1 record). Flowering: June to February; fruiting: August to February.

\section{Millettia macrophylla Benth. - Fig. 2c, d}

Millettia macrophylla Benth. (1848) 788, 789; Hook.f. in Hook.f. \& Benth. (1849) 318, t. 32, 33; Baker (1871) 127; Harms (1909) 68; Dunn (1912) 212; Baker f. (1929) 236; Hepper (1958) 505. - Millettia hookeriana Taub. (1894) 271. - Lectotype (here designated): Vogel 117 (holo K; iso K), Nigeria, 1841.

Tree up to $12 \mathrm{~m}$ high. Twigs hollow, brown strigose. Leaves with 2-6 pairs opposite leaflets. Petiole 2-12.7 cm long, thinly brown strigose, grooved; rachis as the petiole, $5.2-40 \mathrm{~cm}$ long; pulvinus $1-1.5 \mathrm{~cm}$ long, scabrous. Stipules caducous. Leaflets: terminal (narrowly) obovate, $8.4-19.5$ by $4-9.5 \mathrm{~cm}$, index $1.9-3$, base (broadly) cuneate, apex acuminate, acumen 3-10 mm long, upper surface glabrous, lower surface brown strigose, midrib and nerves raised in a furrow or \pm flat above, main nerves 6-11 per side, $12-25 \mathrm{~mm}$ apart, nervation reticulate; lateral mostly as the terminal, obovate, 6.7-23 by $3-8.6 \mathrm{~cm}$, index $1.3-2.8$, base (broadly) cuneate; pulvinus $5-8 \mathrm{~mm}$ long, glabrous. 


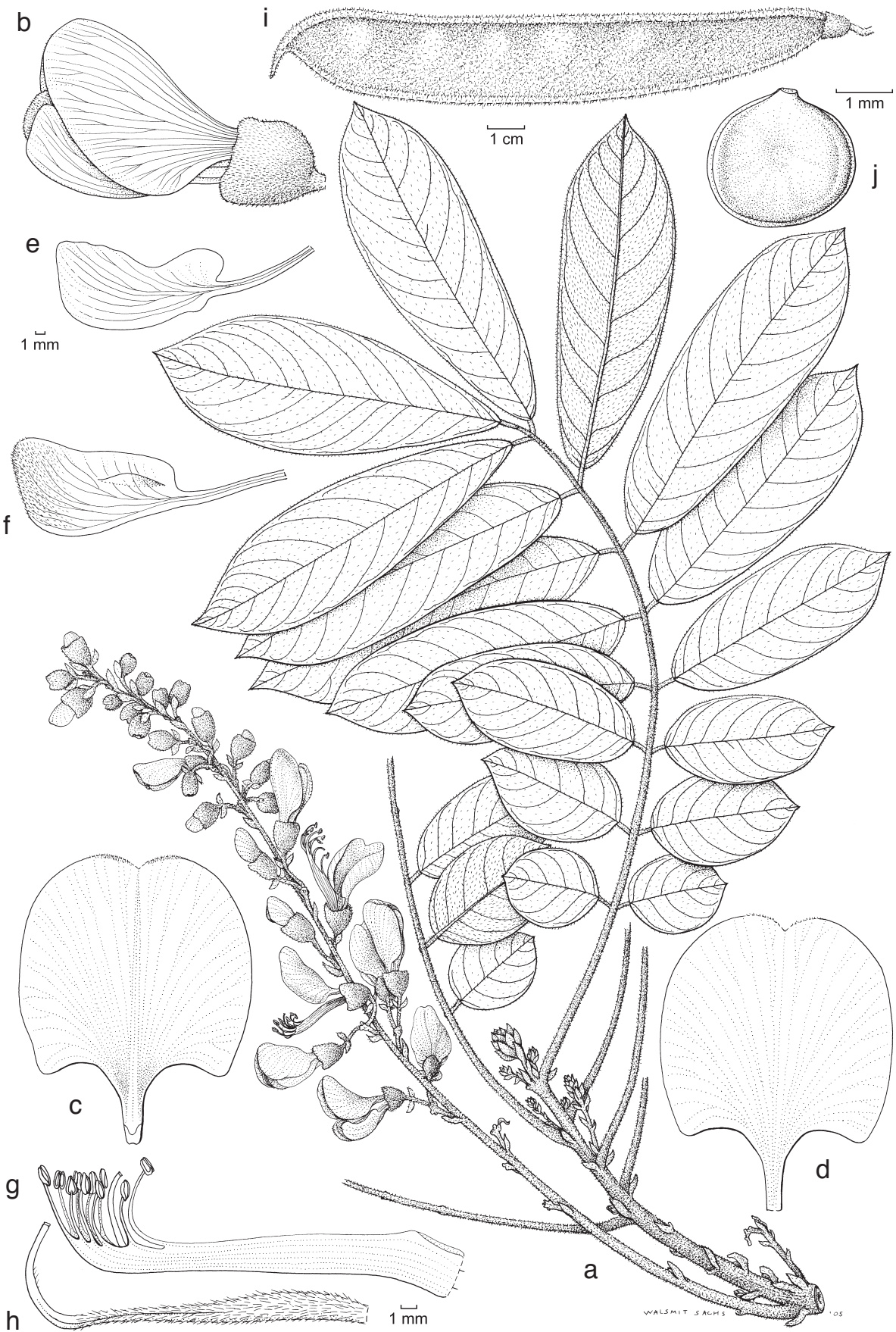

Fig. 1. Millettia aboensis (Hook.f.) Baker. a. Habit; b. flower; c. standard from inside; d. standard from outside; e. wing from outside; f. keel petal from outside; g. stamens; h. pistil; i. pod; j. seed (all: Ansell 360). 


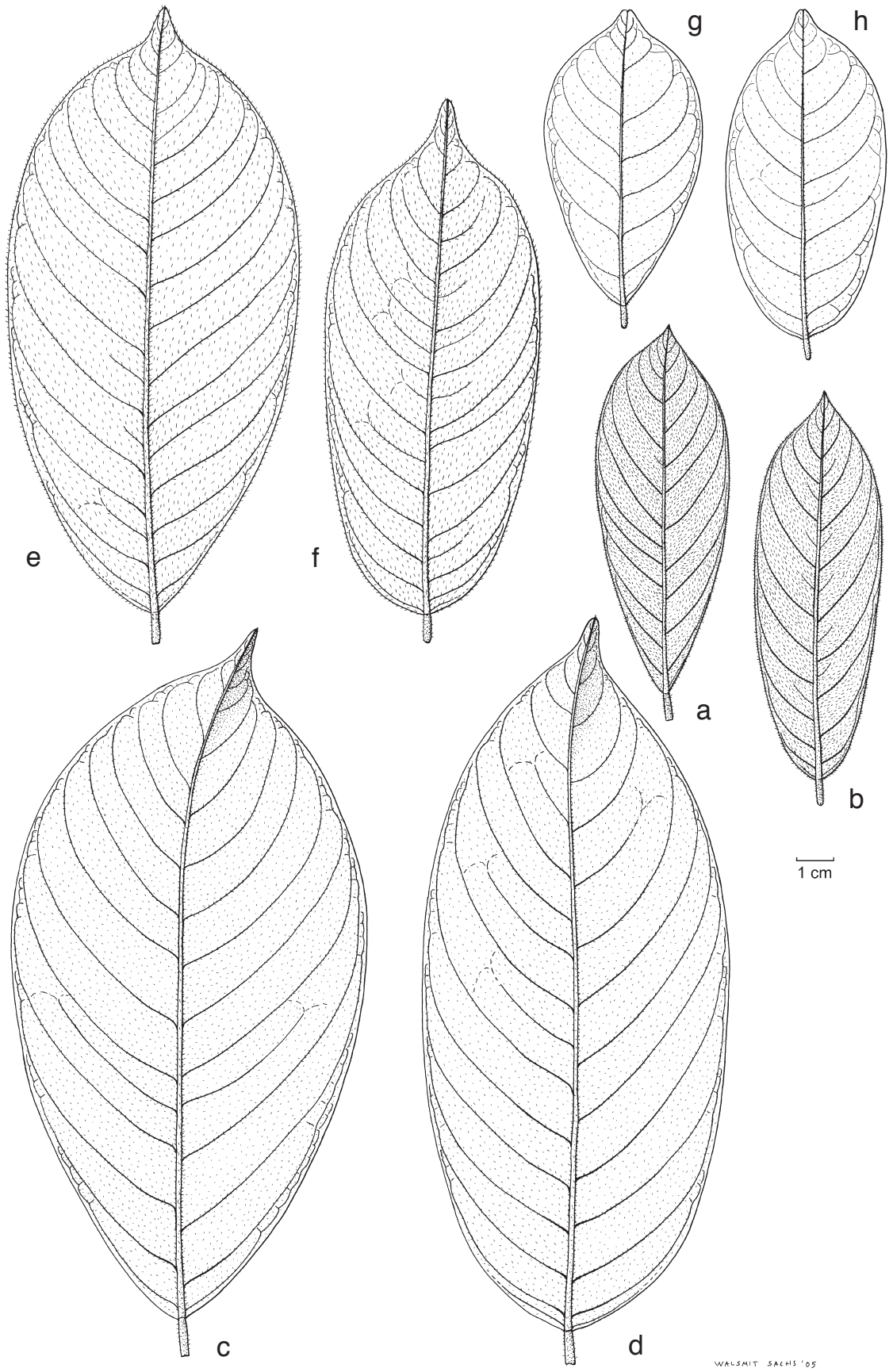

Fig. 2. Millettia Wight \& Arn. sect. Truncaticalyces Dunn. Leaflets from below: a, c, e, g: terminal; b, d, f, h: lateral. a, b. M. aboensis (Hook.f.) Baker; c, d. M. macrophylla Benth.; e, f. M. mannii Baker; g, h. M. thonneri De Wild. (a, b: Ansell 360; c, d: Carvalho 10341; e, f: Champluvier 5110; $\mathrm{g}$, h: Thonner 268). 

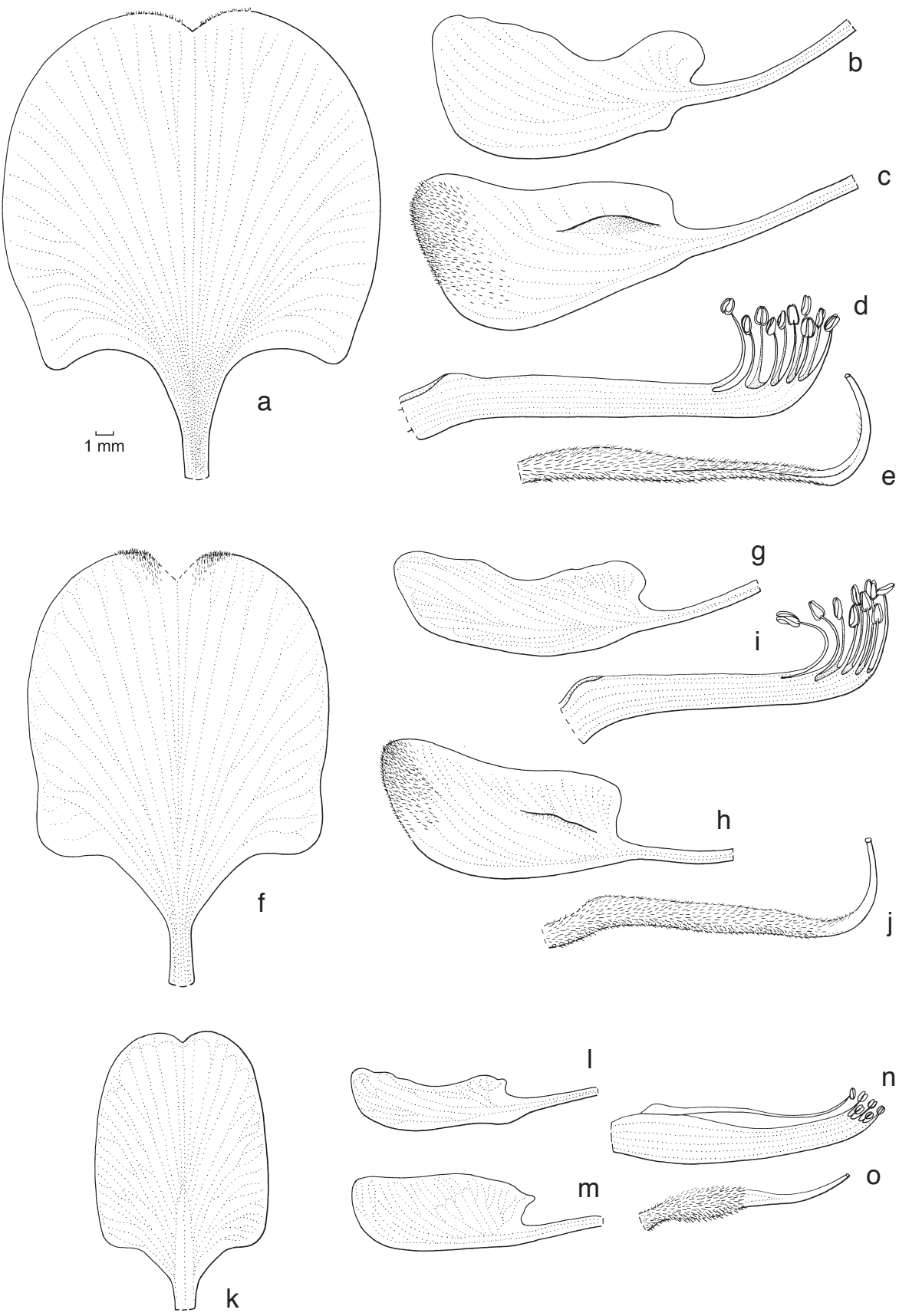

Fig. 3. Millettia Wight \& Arn. sect. Truncaticalyces Dunn. Corolla parts, stamens and pistil of: a-e. M. aboensis (Hook.f.) Baker; f-j. M. mannii Baker; k-o. M. thonneri De Wild. a, f, k. standard from outside; b, g, l. wing from outside; c, h, m. keel petal from outside; d, i, n. stamens; e, j, o. pistil (a-e: Ansell 360; f-j: Champluvier 5110; k-o: Thonner 268). 
Inflorescences 3-35 cm long, brown strigose, peduncle 3-15 cm long, brown strigose; brachyblasts 9-30, c. 8-flowered. Bracts to the inflorescence caducous; other bracts \pm similar, semi-circular, $1-2$ by $0.8-1.8 \mathrm{~mm}$, outside brown sericeous, inside glabrous. Pedicels 3-6 mm long, brown sericeous. Bracteoles at top of pedicel, \pm triangular to semi-circular, $1-1.2$ by $0.8-1.1 \mathrm{~mm}$, outside brown sericeous, inside glabrous. Calyx $3-8 \mathrm{~mm}$ long, truncate or with $0.3-0.5 \mathrm{~mm}$ long, broad-triangular teeth, outside brown sericeous, inside glabrous. Corolla violet to purple. Standard: claw 3-5 mm long; blade broadly ovate or broadly obovate, $13-18$ by $6-15$, apex retuse, mostly glabrous, outside golden sericeous in apical part. Wings: claw 3-9 mm long; blade obliquely ovate or obovate, $11-17$ by $4-5 \mathrm{~mm}$, upper auricle conspicuous, apex obtuse, glabrous. Keel petals: claw $4-10 \mathrm{~mm}$ long; blade hatchet-shaped, $10-14$ by $4-5 \mathrm{~mm}$, lateral pocket 5-7 mm long, apex obtuse, mostly glabrous, outside brown to golden sericeous in apical part. Stamens monadelphous, tube $10-15 \mathrm{~mm}$ long, glabrous, free part of filaments $3-5 \mathrm{~mm}$ long, curved, glabrous; anthers 1.1 by $0.5 \mathrm{~mm}$. Ovary $8-15 \mathrm{~mm}$ long, golden sericeous; ovules 4; style $5 \mathrm{~mm}$ long, curved, strigose at base, glabrous above. Pods 'club'-shaped, flattened, $12-15$ by $3 \mathrm{~cm}$, brown sericeous. Seeds flattened ellipsoid, 10 by $10 \mathrm{~mm}$; hilum c. $3 \mathrm{~mm}$ long.

Distribution - Nigeria, Cameroon, Equatorial Guinea.

Habitat \& Ecology - Mangrove to upland forest. Altitude up to $1100 \mathrm{~m}$. Flowering: June to December; fruiting: September to March.

\section{Millettia mannii Baker - Fig. 2e, f, $3 \mathrm{f}-\mathrm{j}$}

Millettia mannii Baker (1871) 127; De Wild. \& T. Durand (1900) 62; (1907) 147; (1911) 323; Dunn (1912) 212; Baker f. (1929) 236; Hepper (1958) 505. - Type: Mann s.n. (holo K), 1861.

Lonchocarpus zenkeri Taub. (1896) 193. - Type: Zenker (\& Staudt) 100 (holo K), Cameroon, 1896. Millettia aboensis (Hook.f.) Baker var. glabrescens Dunn (1912) 214; Baker f. (1929) 236. - Type: Zenker \& Staudt 100 (holo K), Cameroon, Yaunde.

Tree up to $11 \mathrm{~m}$ high. Bark smooth, dark brown. Stems hollow, brown strigose. Leaves with 7-11 pairs opposite leaflets. Petiole 5-12 cm long, thinly brown strigose; rachis $16-39 \mathrm{~cm}$ long, brown strigose; pulvinus $0.5-1.5 \mathrm{~cm}$ long, strigose. Stipules caducous. Leaflets: terminal (narrowly) ovate, $5.5-17.5$ by $2.8-7 \mathrm{~cm}$, index $1.7-2.7$, base acute to cuneate, apex acuminate, acumen 3-15 mm long, upper surface scabrous, lower surface sericeous, midrib and nerves sunken, main nerves $6-13$ per side, $6-25 \mathrm{~mm}$ apart, nervation reticulate; lateral mostly as the terminal, obovate, $3.5-10$ by $2.5-5.5$ $\mathrm{cm}$, index 1.4-2.5, base (broadly) acute; pulvinus 3-10 $\mathrm{mm}$ long, brown strigose. Inflorescences 8-47.5 cm long, brown sericeous, peduncle 4-27 cm long, brown sericeous; brachyblasts c. 30, 7-10-flowered. Bracts to the inflorescence linear-ovate $5-7$ by $1-2 \mathrm{~mm}$, both sides sericeous; other bracts \pm similar, semi-circular $1-1.5$ by 1-1.5 cm, outside brown sericeous, inside glabrous. Pedicels $3-4 \mathrm{~mm}$ long, brown sericeous. Bracteoles at top of pedicel, \pm triangular to semi-circular, 1-2 mm long, outside sericeous, inside glabrous. Calyx 5-7 mm long, truncate, outside brown sericeous, inside glabrous. Corolla red violet to purple. Standard: claw 3-8 $\mathrm{mm}$ long; blade broadly ovate to broadly obovate, $10-20$ by $10-18 \mathrm{~mm}$, apex retuse to emarginate, mostly glabrous, outside golden sericeous in apical part. Wings: claw 3-11 mm long; blade obliquely ovate, narrower at the centre or clavate, $13-17$ by $2-4 \mathrm{~mm}$, apex 
obtuse, upper auricle conspicuous, both sides glabrous. Keel petals: claw 3-8 mm long; blade hatchet-shaped, $10-16$ by $4-5 \mathrm{~mm}$, apex obtuse, lateral pocket $2-5 \mathrm{~mm}$ long, mostly glabrous, outside brown to golden sericeous in apical part. Stamens monadelphous, white, purple tinged, tube 10-20 mm long, glabrous, free part of filaments $3-7 \mathrm{~mm}$ long, glabrous; anthers pale yellow, $1-1.2$ by $0.1-1 \mathrm{~mm}$. Ovary $10-22 \mathrm{~mm}$ long, golden sericeous; ovule 3-8; style pale green, 3-10 mm long, curved, strigose at base, glabrous above. Pods strap- to 'club'-shaped, \pm narrower at base than at apex, flattened, $11-15$ by $1.2-2.2 \mathrm{~cm}$, brown sericeous. Seeds flattened ellipsoid, 10-13 by 9-13 mm, hilum 1-5 mm long.

Distribution - Cameroon, Gabon, Congo.

Habitat \& Ecology - Secondary disturbed forest. Altitude 200-700 m. Flowering: August to April; fruiting: October to April.

Note - Dunn annotated the Kew specimen of Zenker \& Staudt 100 as the type of his var. glabrescens of M. aboensis. Another duplicate of this collection, cited as Zenker 100, was used by Taubert (1896) to describe Lonchocarpus zenkeri. As this specimen (Zenker (\& Staudt) 100) belongs to M. mannii, both names are synonyms of M. mannii.

\section{Millettia psilopetala Harms}

Millettia psilopetala Harms (1911) 256; Dunn (1912) 213; Baker f. (1929) 235; Robijns (1948) 314; Eggeling (1952) 307, t. 1; Hauman (1954) 20, t. 1; J.B. Gillett (1971) 19. - Lectotype (Gillett, 1971): Mildbraed 2290 (holo B †; iso BR, K, drawing and fragments), E Congo, Beni, 1908.

Craibia bequaerti De Wild. (1925) 362; Baker f. (1929) 247. - Lectotype (here designated): Bequaert 6647 (holo BR), Congo, 1915.

Lianas up to $12 \mathrm{~m}$ high. Twigs brown strigose. Leaves with 2-6 pairs opposite or alternate leaflets. Petiole $0.3-12 \mathrm{~cm}$ long, thinly brown strigose; rachis $0.4-28 \mathrm{~cm}$ long, thinly brown strigose; pulvinus $0.5-1 \mathrm{~cm}$ long, glabrous. Stipules caducous. Leaflets: terminal (narrowly) obovate, $7.3-19$ by $2.3-8 \mathrm{~cm}$, index $1.7-3.3$, base acute to cuneate, apex acuminate to cuspidate, acumen $0.5-3 \mathrm{~cm}$ long, upper surface scabrous to glabrous, lower surface scabrous, midrib and nerves sunken, main nerves 4-9 per side, 8-25 mm apart, nervation reticulate; lateral mostly as the terminal (broadly) obovate, $4-7.8$ by $3.8-4.8 \mathrm{~cm}$, index 1.1-2.5, base (broadly) acute; pulvinus $5-12 \mathrm{~mm}$ long, brown strigose. Inflorescences $13-47 \mathrm{~cm}$ long, brown strigose, peduncle up to $8.5 \mathrm{~cm}$ long, brown strigose; brachyblasts 20 to many, 10- to many-flowered. Bracts \pm similar, triangular, 0.5-5 mm long, outside brown sericeous, inside glabrous. Pedicels 3-4 $\mathrm{mm}$ long, brown strigose. Bracteoles \pm triangular, 1-2 mm long, outside sericeous, inside glabrous. Calyx 4-5 mm long, truncate or with $0.2-1.5 \mathrm{~mm}$ long broad-triangular teeth, outside thinly brown strigose, inside glabrous. Corolla purple to magenta. Standard: $2-3 \mathrm{~mm}$ long; blade broadly ovate, $6-10$ by $6-10 \mathrm{~mm}$, apex retuse, both sides glabrous. Wings: claw 2-4 mm long; blade narrowly ovate, $7-10$ by $2-4 \mathrm{~mm}$, apex obtuse, auricles conspicuous, both sides glabrous. Keel petals: claw 3-5 mm long; blade narrowly obovate or elliptic, $7-10$ by $2-4 \mathrm{~mm}$, upper auricle conspicuous, lateral pocket 1-4 mm long, both sides glabrous. Stamens diadelphous, tube 2-9 mm long, glabrous, free part of filaments $2-4 \mathrm{~mm}$ long, glabrous; anthers $0.4-1.5$ by $0.2-1.3 \mathrm{~mm}$. Ovary 2-8 mm long, sericeous; ovules 3 or 4; style 2-4 mm long, curved or bending 
towards the ovary, glabrous. Pods strap-shaped, flattened, sometimes narrower at the centre, $12-13$ by $3.3-3.5 \mathrm{~cm}$, brown sericeous. Seeds flattened ellipsoid, black, 18 by $20 \mathrm{~mm}$; hilum $10 \mathrm{~mm}$ long.

Distribution - RD Congo, Burundi, Rwanda, Uganda.

Habitat \& Ecology - Moist forest. Altitude 470-800 m. Flowering: throughout the year; fruiting: October to June.

\section{Millettia thonneri De Wild. - Fig. $2 \mathrm{~g}$, h, 3k-o}

Millettia thonneri De Wild. (1911) 218, t. 1; Dunn (1912) 213; Baker f. (1929) 235. - Lectotype (here designated): Thonner 268 (holo K; iso BR, K), Congo, 16.01.1909.

Tree c. $3 \mathrm{~m}$ high. Twigs hollow, brown strigose. Leaves with 1-4 pairs opposite leaflets. Petiole 4.5-16 cm long, grooved, thinly brown strigose; rachis as the petiole, $2-9 \mathrm{~cm}$ long; pulvinus $0.8-1 \mathrm{~cm}$ long, scabrous. Stipules caducous. Leaflets: terminal (broadly) obovate, $7.5-9.5$ by $3.5-5.5 \mathrm{~cm}$, index $1.7-2.1$, base acute, apex acuminate, acumen, 5-6 mm long, retuse, upper surface glabrous, lower surface with few appressed hairs along midrib and some scattered appressed hairs elsewhere, midrib and nerves flat or \pm sunken, main nerves $7-9$ per side, $10-15 \mathrm{~mm}$ apart, nervation reticulate; lateral mostly as the terminal, obovate, $5-10.5$ by $3.5-5 \mathrm{~cm}$, index $1.4-2$; pulvinus $8-10 \mathrm{~mm}$ long, thinly strigose. Inflorescences $12-24 \mathrm{~mm}$ long, brown strigose, peduncle 11-19.5 cm long, brown strigose; brachyblasts many. Bracts to the inflorescences caducous; other bracts \pm similar, semi-circular, 1 by $1 \mathrm{~mm}$, outside brown sericeous, inside glabrous. Pedicels 2-4 mm long, brown sericeous. Bracteoles at top of pedicel, semi-circular, 1 by $1 \mathrm{~mm}$, outside sericeous, inside glabrous. Calyx $2-3 \mathrm{~mm}$ long, truncate or with $0.5 \mathrm{~mm}$ long broad-triangular teeth, outside brown sericeous, inside glabrous. Corolla purple. Standard: claw $2-3 \mathrm{~mm}$ long; blade broadly obovate, $12-13$ by $8-10 \mathrm{~mm}$, apex retuse, both sides glabrous. Wings: claw $5 \mathrm{~mm}$ long; blade obliquely ovate, 9-10 by $2 \mathrm{~mm}$, apex obtuse, upper auricle conspicuous, both sides glabrous. Keel petals: claw $5 \mathrm{~mm}$ long; blade hatchet-shaped 10 by $3 \mathrm{~mm}$, apex obtuse, both sides glabrous. Stamens diadelphous, tube 12-18 mm long, glabrous, free part of filaments $2 \mathrm{~mm}$ long, curved, glabrous; anthers 0.3 by $0.3 \mathrm{~mm}$. Ovary $7-10 \mathrm{~mm}$ long, inflated, brown sericeous; ovules 6; style 4-5 mm long, curved near the stigma, glabrous.

Distribution - RD Congo.

Habitat \& Ecology - Rare, once collected. River bank. Altitude 410 m. Flowering: March.

Note - Millettia thonneri is known from only one collection with in total five sheets in the Brussels and Kew herbaria. Apparently it has not been collected again since 1909. However, we have not seen material from African herbaria, so we are not totally certain whether or not the species still can be found. But as Kew and Wageningen receive duplicates from most African collections, this species is probably not collected again. It is impossible to ascertain its conservation status, or propose strategies for its conservation. A search at the original locality or similar habitats is needed to ascertain its present-day status. 


\section{ACKNOWLEDGEMENTS}

The loan of material from BR, K, L, WAG used in this study is highly appreciated. The first author likes to thank the IFP-Ford Foundation for financial support and Dr. M.C. Roos (L), coordinator of the MSC program.

\section{REFERENCES}

Allen, O.N. \& E.K. Allen. 1981. The Leguminosae. A source book of characteristics, uses and nodulation. MacMillan Publishers Ltd., London.

Baker, E. G. 1929. The Leguminosae of Tropical Africa. Part 2. Unitas Press, Ostend.

Baker, J.G. 1871. Leguminosae. Suborder I. Papilonaceae. In: D. Oliver, Flora of Tropical Africa 2: 1-258. Reeve \& Co, London.

Baker, J.G. 1879. Leguminosae. In: J.D. Hooker, Flora of British India 2: 56-306. Reeve \& Co, London.

Bentham, G. 1848. Millettia macrophylla Benth. In: W.J. Hooker, Icones Plantarum 7: t. 788, 789.

Bentham, G. 1852. Leguminosae. In: F. A.W. Miquel, Plantae Junghuhnianae: 205-269. Sythoff, Leiden.

De Wildeman, E. 1907. Etudes de systématique et de Géographie botaniques sur la Flore du Bas- et du Moyen-Congo. Ann. Mus. Congo Belge, Bot., Ser. 5, 2: 1-368.

De Wildeman, E. 1911. Etudes sur la flore des districts de Bangala et de l'Ubangi (Congo, Belge). Misch \& Thron, Bruxelles.

De Wildeman, E. 1925. Plantae Bequaertianae. 3. A. Buyens, Gand; J. Lechevalier, Paris.

De Wildeman, E. \& Th. Durand. 1900. Reliquiae Dewevreanae. Ann. Mus. Congo Belge, Bot., Ser. 3, 1: 1-287.

Dunn, S.T. 1912. A revision of the genus Millettia Wight et Arn. J. Linn. Soc., Bot. 41: 123-243.

Eggeling, W.J. 1952. Indigenous trees of the Uganda Protectorate. Ed. 2. Crown Agents, London.

Geesink, R. 1981. Tribe 6. Tephrosieae (Benth.) Hutch. (1964). In: R.M. Polhill \& P.H. Raven (eds.), Advances in Legume Systematics 1: 245-260. Royal Botanic Gardens, Kew.

Geesink, R. 1984. Scala Millettiarum. Leiden Bot. Ser. 8.

Gillett, J.B. 1971. Tephrosieae. In: E. Milne-Redhead \& R.M. Polhill (eds.), Flora of Tropical East Africa. Part 3, 1. Crown Agents, London.

Harms, H. 1909. Einige Nutzhölzer Kameruns. II. Leguminosae. Notizbl. Bot. Gart. Berlin-Dahlem, Append. 21: 9-75.

Harms, H. 1911. Leguminosae. In: J. Mildbraed, Wissenschaftliche Ergebnisse der Deutschen ZentralAfrika-Expedition 1907-1908, 2, Botanik: 232-270. Klinkharat \& Bierman, Leipzig.

Hauman, L. 1954. Flore du Congo Belge et du Rwanda-Urundi, 5: 5-51. Bruxelles.

Hepper, F.N. 1958. Papilionaceae. In: R.W.J. Keay (ed.), Flora West Tropical Africa, 2nd ed. Crown Agents, London.

Hooker, J.D. \& G. Bentham. 1849. Leguminosae. In: W.J. Hooker, Niger Flora: 291-332. Hippolyte Bailliere, London.

Hutchinson, J. 1964. The genera of flowering plants. Clarendon Press, Oxford.

Robijns, W. 1948. Flore des Spermatophytes du Parc National Albert. 1. Bruxelles.

Taubert, P. 1894. Leguminosae. In: A. Engler \& A. Prantl, Die natürlichen Pflanzenfamilien 3, 3: 70-388.

Taubert, P. 1896. Leguminosae africanae. I. Bot. Jahrb. Syst. 23: 172-196.

Wight, R. \& G.A.W. Arnott. 1834. Prodromus Florae Peninsulae Indiae orientalis. 


\section{IDENTIFICATION LIST}

The numbers after the collection numbers refer to the following species:

$$
\begin{aligned}
& 1=M \cdot \text { aboensis } \\
& 2=M \cdot \text { macrophylla } \\
& 3=M \cdot \text { mannii } \\
& 4=M \cdot \text { psilopetala } \\
& 5=M . \text { thonneri }
\end{aligned}
$$

Akim 33837: 1 - Ansell 360: 1

Bamps 1720: 3 - Bates 59a: 2; 59b: 3 - Binuyo 41439: 2 - Boss 3004: 3; 4284: 3 - Breteler 829: $3 ; 2690: 3 ; 8415: 3 ; 8670: 3$.

Cambridge 261: 4 - Carvalho 10341: 2 - Champluvier 5110: 3; 5265: 3; 5266: 3; 5288:3 - Chizea 24477: 1 - Christiaensen 911: 4; 1829: 4.

Daramola 55180: 1 - J. de Wilde 11: 4; 33: 4; 582: 4 - W. de Wilde 1222: 3; 2015: 3; 3834: 3; 3897: 3; 7848: 3 - De Witte 1074: 4; 7945: 4; 13010: 4 - Dennett 15: 1.

Eilu 327: 4; 389: 4 - Ewango 569: 4.

Gilbert 7981: 4; 9389: 4 - Gille 231: 4.

Hart 481: 4; 617: 4; 781: 4; 902: 4.

Jans 814: 4.

Latilo 67613: 1; 67681: 1 - Le Testu 7303: 3; 8170: 3 - Lebrun 4191: 4 - Leeuwenberg 8963: 3; 9041: 3 - Lewalle 3972: 4 - Liengola 213: 4; 214: 4 - Lingola 31A: 4 - A.M. Louis 829: 3; 1270: 3 - J. Louis 6632: 4; 7505: 4; 16420: 4.

Marabo 1451: 4 - McPherson 13812: 3 - Meijer 15048: 3.

Okafor 57608: 1 - Olorunfemi 43862: 1 - Onochie 34054: 1.

Purseglove 3419: 4.

Runyinya 327: 4.

Satabié 318: 2; 324: 2.

Talbot 1345: 1 - D. Thomas 3500: 1 - D.W. Thomas 2334: 2 - N.W. Thomas 1664: 1 - Thonner 268: 5 - Troupin 4473: 4; 9339: 4; 12080: 4; 12131: 4; 12599: 4; 13529: 4; 13576: 4; 15710: 4.

Van der Veken 9734: 4 - Vogel 117: 2.

Zenker 100: 3 ; 1173: 3; 3110: 2; 4314: 2 . 